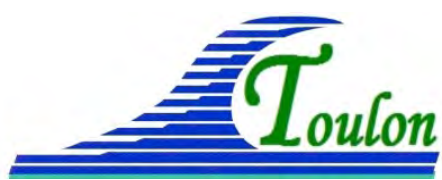

\title{
Analyse des mécanismes d'érosion le long du passage du Gois, Vendée
}

\author{
Baptiste LE MAUFF ${ }^{1}$, Benoît WAELES ${ }^{2}$
}

1. Université de Nantes, IGARUN, UMR 6554 CNRS - LETG, Campus du Tertre, BP 81 227, 44312 Nantes, France. baptiste.le-mauff@univ-nantes.fr

2. Consultant Génie Côtier, 53 rue du commandant Groix, 29200 Brest, France. benoit.waeles@gmail.com

\section{Résumé :}

Le Passage du Gois est une chaussée submergée à chaque marée. Il permet de relier le continent à l'île de Noirmoutier à marée basse en traversant la Baie de Bourgneuf. L'érosion constatée le long de la chaussée côté Sud remet en cause sa pérennité. Une analyse des processus hydro-sédimentaires à l'origine de cette érosion a été réalisée pour la DREAL Pays-de-la-Loire dans le cadre d'un projet de classement du site.

Le Gois est à l'origine une édification sédimentaire issue d'un hydrodynamisme particulier dans la baie. L'onde de marée pénètre dans celle-ci par ses deux ouvertures : une ouverture étroite au Sud (le goulet de Fromentine) et une ouverture plus large au Nord entre la pointe Nord de Noirmoutier et le continent. La rencontre des deux courants de flots a favorisé une zone d'accumulation sableuse dans la partie Sud de la Baie. Le tracé du Gois a été fixé (pavage) au début du $20^{\text {ème }}$ siècle alors que les conditions hydrodynamiques évoluent en même temps que la morphologie de la Baie de Bourgneuf. Celle-ci a été largement aménagée: endiguement du trait de côte et diminution de la surface de la baie. Les conditions hydrodynamiques actuelles sont analysées à l'aide d'un modèle hydrodynamique (Mike21 HD développé par DHI) notamment validé par des mesures de courant et de niveau d'eau (ADV Océan $5 \mathrm{MHz}$ ) dans le chenal qui longe la partie Sud du Passage du Gois. Les schémas de transport sédimentaire sont ensuite simulés avec un modèle hydro-sédimentaire (Mike21 ST). Les résultats des modèles et l'analyse des évolutions morphologiques passées montrent que le courant de flot, qui traverse la chaussée depuis le Nord de la baie de Bourgneuf, est un facteur essentiel de l'érosion au Sud de l'ouvrage.

Mots-clés : Modélisation hydro-sédimentaire, Evolution morphologique, Ouvrage, Baie de Bourgneuf.

\section{Introduction}

Le Passage du Gois est une route submersible longue de $4.2 \mathrm{~km}$ qui relie le continent, depuis Beauvoir-sur-mer (85), à l'île de Noirmoutier à marée basse. Il est à l'origine un haut fond sableux mentionné pour la première fois dans des écrits autour de 830. Il est 


\section{Thème 2 - Dynamique sédimentaire}

cartographié pour la première fois par Claude Masse en 1702 (SUIRE, 2011). On constate depuis lors que son tracé évolue (COQUET et al., 2014). Afin de permettre la traversée en voiture, le Gois est pavé pendant la première moitié du $20^{\text {ème }}$ siècle: son tracé est définitivement fixé. Bien qu'il ne soit plus l'accès principal à l'île par la route (le pont de Noirmoutier est construit au-dessus du Goulet de Fromentine en 1971), le Passage du Gois demeure un patrimoine historique et naturel unique inscrit à l’inventaire des sites nationaux. La DREAL Pays-de-la-Loire a récemment engagé une procédure pour le classement du site. Cependant une érosion de la chaussée est constatée côté Sud. Une marche entre le flanc de la chaussée et le bord du chenal adjacent peut atteindre 1m sur certains secteurs (COQUET et al., 2014). Cette érosion remet en cause la pérennité de l'ouvrage. La DREAL souhaite qu'un aménagement soit mis en place pour contenir l'érosion sans modifier l'apparence historique de la chaussée. Cette étude vise à comprendre les processus régissant la dynamique sédimentaire aux abords du Passage du Gois afin d’identifier les mécanismes à l'origine de l'érosion constatée sur la partie Sud de l'ouvrage.

\section{Caractérisation du site d'étude}

\subsection{Contexte et évolution géomorphologique}

La Baie de Bourgneuf est une baie relativement peu profonde de 60000 ha qui se situe au Sud de l'estuaire de la Loire (figure 1). Abritée derrière une île barrière (l'île de Noirmoutier), deux ouvertures permettent le remplissage et la vidange de la baie pendant la marée. Cependant, l'ouverture Nord est large d'environ $12.5 \mathrm{~km}$, alors que l'ouverture Sud (le Goulet de Fromentine) est une embouchure tidale large de moins de 500m (figure 1). Le Passage du Gois tient son origine naturelle d'un hydrodynamisme particulier lié à cette configuration de la baie et à la marée qui s'y propage. L'onde de marée pénètre la baie de Bourgneuf par ses deux ouvertures. La convergence des flux lors de leur rencontre favorise une zone d'accumulation sableuse et l'édification progressive d'un isthme naturel découvert à marée basse (VERGER, 2009 ; BERNIER \& GRUET, 2011). La baie est divisée en deux versants de part et d'autre du Gois. Le versant Sud, très peu profond, a la forme d'un entonnoir qui débouche sur une embouchure tidale : le Goulet de Fromentine (figure 1). Cette partie de la baie est incisée d'un système de chenaux développés qui semblent être en partie le prolongement du delta de flot de l'embouchure tidale.

A partir de l'étude de cartes anciennes, de plusieurs levés bathymétriques et d'images aériennes, deux études retracent l'évolution morphologique du versant Sud de la Baie de Bourgneuf depuis environ deux siècles. La surface du versant Sud de la baie a diminué progressivement à cause de la poldérisation (COQUET et al., 2014). Le trait de côte est endigué presque intégralement à l'exception du Goulet de Fromentine. Depuis 1822, La largeur de ce dernier a diminué de $200 \mathrm{~m}$ et sa profondeur maximale a augmenté 


\section{XIV èmes Journées Nationales Génie Côtier - Génie Civil \\ Toulon, 29 juin au $1^{\text {er }}$ juillet 2016}

d'environ 7m, la section mouillée ayant diminué de 30\% (CREOCEAN, 2011). D’après la relation empirique d'O’Brien (O’BRIEN, 1969), qui relie la section mouillée minimale d'une embouchure au prisme de marée, cette diminution pourrait impliquer une diminution du prisme de marée se traduisant par une pénétration plus faible du flot par l'ouverture Sud.

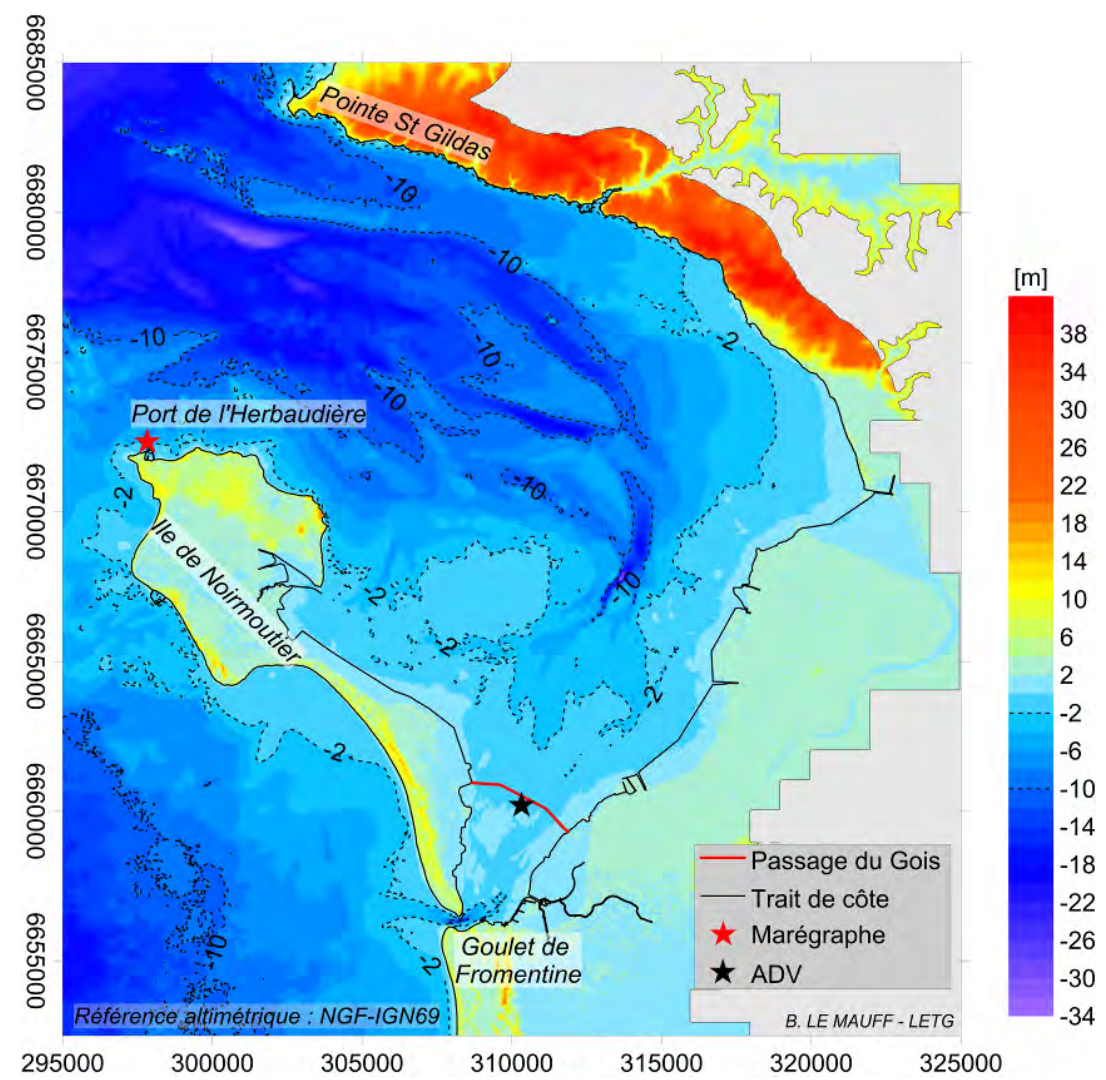

Figure 1. Topo-bathymétrie du site d'étude et position du marégraphe de l'Herbaudière et de l'ADV déployé dans le cadre de l'étude.

Des images aériennes et satellitaires ont été rassemblées de 1945 à nos jours depuis le portail web public Géoportail (figure 2). On constate des tendances à l'érosion côté Sud de la chaussée sans que l'on y observe un chenal continu comme c'est le cas actuellement. Plusieurs chenaux relativement perpendiculaires au Passage se créent ; leur orientation (S-SW) semble indiquer une érosion liée au courant de flot provenant du Nord qui traverse la chaussée et provoque une tendance à l'érosion en aval de celleci. Il se pourrait que le Passage du Gois se trouve actuellement dans une zone de flux solide résiduels orientés vers le Sud. La mise en place du modèle hydro-sédimentaire va nous permettre d'analyser cette hypothèse. 


\section{Thème 2 - Dynamique sédimentaire}

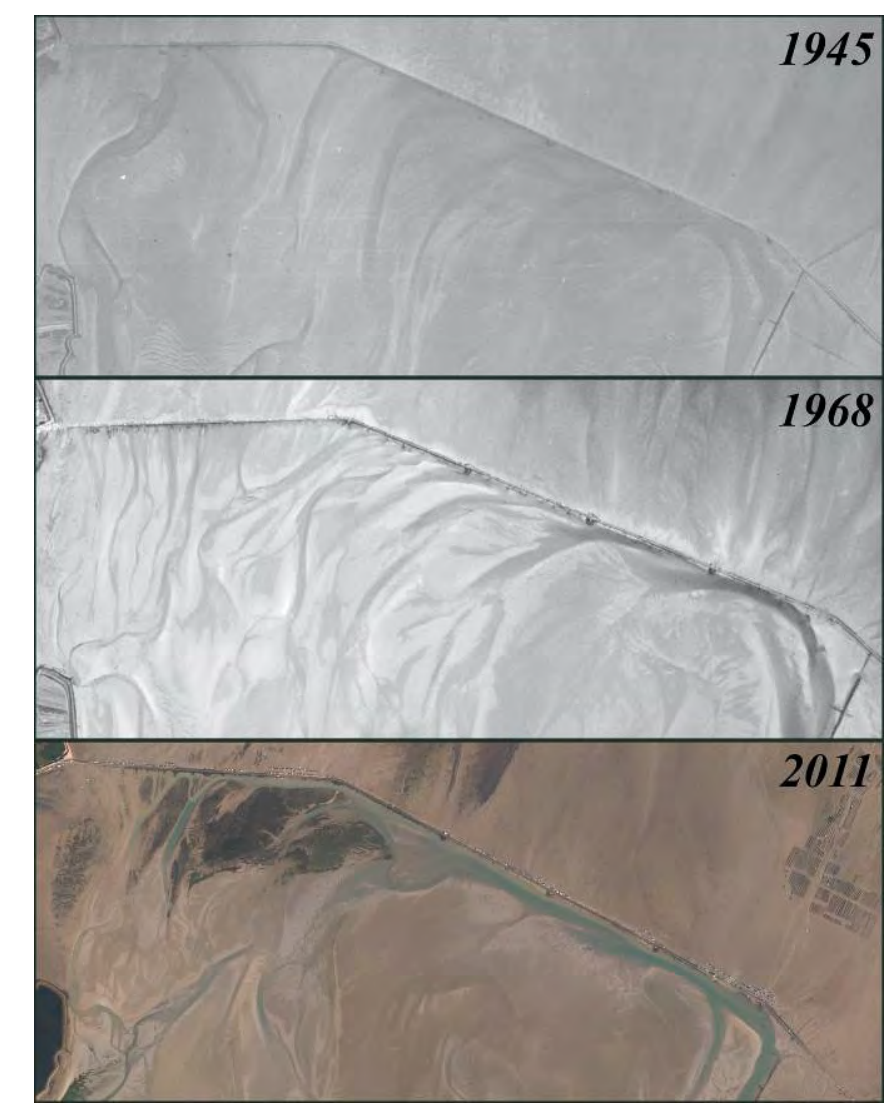

Figure 2. Images aériennes du Passage du Gois de 1945, 1968 et 2011.

Une modification de la configuration morphologique de la baie provoque une modification des conditions hydrodynamiques. Si la rencontre des deux courants de flot de directions opposées s'effectue plus au sud qu'originellement, on peut faire l'hypothèse que la zone d'accumulation sableuse devrait se déplacer vers le Sud (BERNIER \& GRUET, 2011).

\subsection{Contexte hydrodynamique}

Le marnage dans la Baie de Bourgneuf varie d'environ $2 \mathrm{~m}$ en morte-eau à $6 \mathrm{~m}$ en viveeau (régime macro-tidal). Les deux ouvertures de la Baie de Bourgneuf induisent un cycle de marée particulier. Lors du flot, les volumes d'eau passant par la large ouverture Nord sont plus importants que par l'étranglement du Sud.

La baie de Bourgneuf est bien abritée derrière l'île de Noirmoutier de la houle incidente au large généralement de secteur ouest. La réfraction, le frottement et le déferlement des vagues en raison des faibles profondeurs de la baie atténuent largement leur énergie.

La circulation des masses d'eau et les vitesses induites à l'approche du Gois sont précisées par des mesures hydrodynamiques et la mise en place d'un modèle dédié décrits ci-après. 


\section{XIV èmes Journées Nationales Génie Côtier - Génie Civil \\ Toulon, 29 juin au $1^{\text {er }}$ juillet 2016}

\subsection{Les mesures de courants et de hauteur d'eau au Passage du Gois}

Aucune mesure de courant n’a été identifiée dans ce secteur. Le dispositif léger déployé pour l'étude permet d'apporter un premier élément de calage pour la mise en place d'un modèle hydrodynamique. L'appareil de mesure utilisé pour cette campagne est un ADV (Acoustic Doppler Velocimeter) Ocean fabriqué par SONTEK et appartenant au laboratoire LETG. La direction et la vitesse instantanées des courants ont été mesurées ainsi que les variations de niveau d'eau. Les courants de marée étant dominants dans la zone, nous avons donc mesuré un cycle de marée complet (coefficient 95) pendant la nuit du 16 au 17 avril 2015. L'ADV a été placé dans le chenal bordant la rive sud de la chaussée entre la 5ème et la 6ème balise en partant de Noirmoutier. L'appareil a été positionné de sorte que les mesures soient perturbées le moins possible par des turbulences liées à la présence de la chaussée, des balises et des déflecteurs.

On constate deux inflexions dans la pente de la courbe de marée entre PM-4h et PM-3h puis entre $\mathrm{PM}+3 \mathrm{~h}$ et $\mathrm{PM}+4 \mathrm{~h}$ (figure 3) correspondant à la cote du Passage du Gois. On distingue quatre phases dans le schéma de circulation (figure 3) dont il faut retenir que le flot du Nord renverse le flot du Sud avant même que le niveau de pleine mer ne soit atteint.
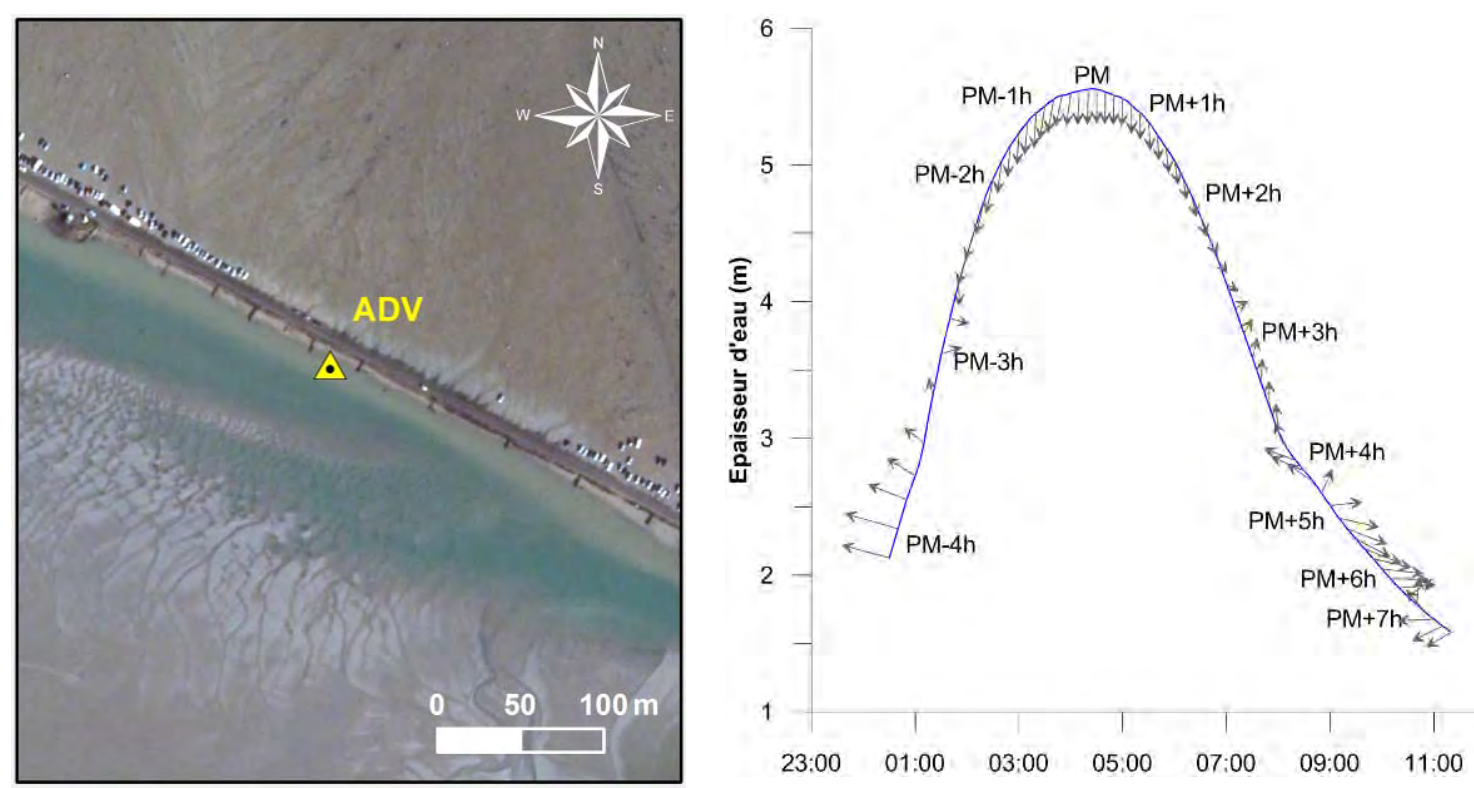

Figure 3. Les mesures de l'ADV déployé le 17/04/2015 dans le chenal au Sud de la chaussée : courbe de marée (en bleu), vitesse et intensité des courants (vecteurs).

\section{Modélisation des flux sédimentaires}

\subsection{Configuration du modèle}

Les simulations sont effectuées à l'aide du logiciel MIKE21 développé par le Danish Hydraulic Institute (DHI). Ce code résout par une méthode d'éléments finis sur des 


\section{Thème 2 - Dynamique sédimentaire}

maillages triangulaires, les équations de l'hydraulique pour les écoulements à surface libre en deux dimensions. Les courants calculés par le modèle hydrodynamique (Mike21 HD) constituent les variables forçantes du modèle de transport sédimentaire (Mike21 ST). La modélisation du transport a été réalisée sur le même domaine et le même maillage que les simulations hydrodynamiques. Les paramètres de hauteur d'eau, vitesse et direction de courant ainsi que de capacité de transport solide (en magnitude et direction) sont notamment calculés sur un cycle de marée de vive-eau (coefficient 95) correspondant aux mesures hydrodynamiques présentées précédemment. Le transport de sable est simulé en chaque point du maillage par une formule de capacité de transport total (charriage + suspension) appliquée à des sédiments de granulométrie déterminée. La répartition granulométrique des sables est supposée constante et la disponibilité en sable est supposée totale sur l'ensemble du domaine.

Le domaine de calcul s'étend depuis la Baie de Concarneau à La Tranche sur Mer et jusqu'à $130 \mathrm{~km}$ au large. Une gradation de la définition du maillage permet d'optimiser le rapport entre une bonne représentation des phénomènes physiques et des temps de calcul raisonnables. Les mailles les plus fines (environ $2 \mathrm{~m}$ à $5 \mathrm{~m}$ ) se trouvent sur le passage du Gois afin de bien représenter l'obstacle à l'écoulement dans le modèle (figure 4).

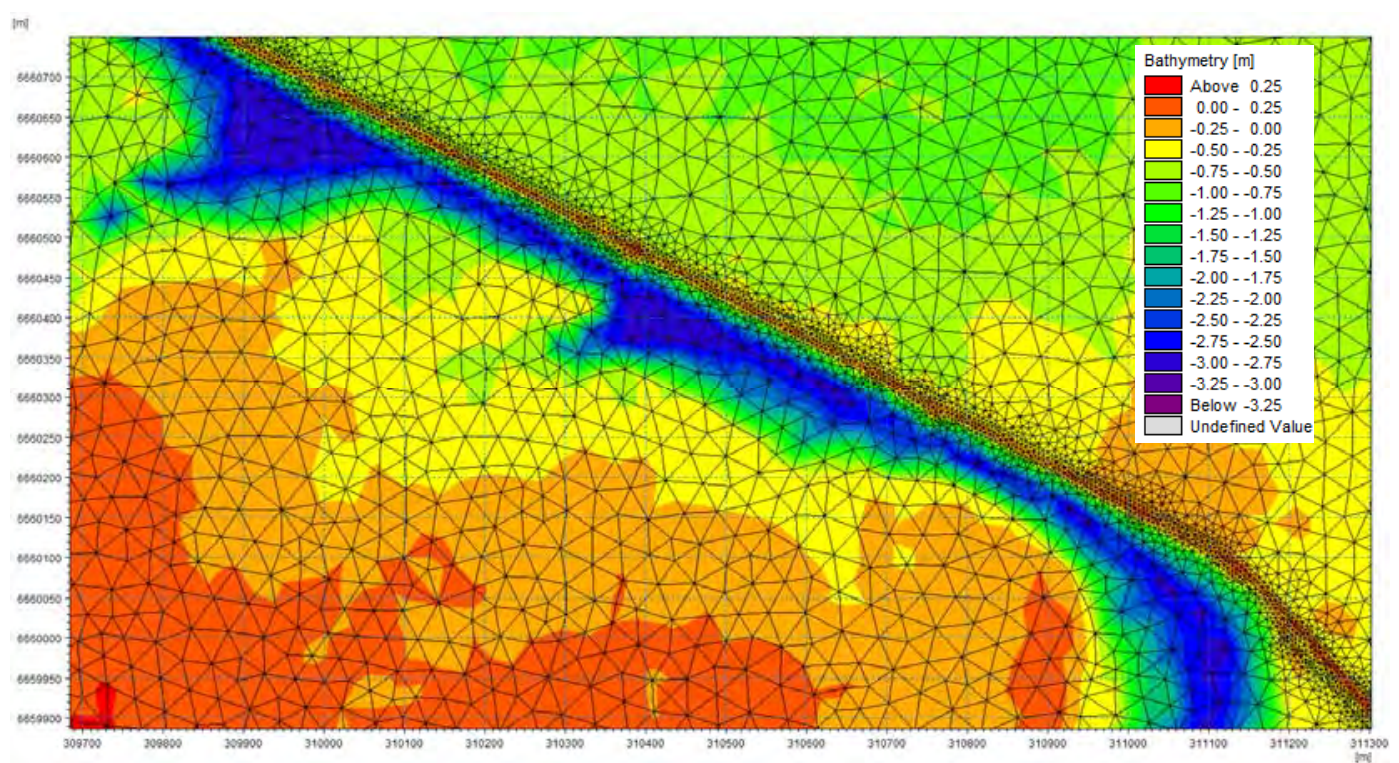

Figure 4. Le maillage du domaine de calcul aux abords du Passage du Gois.

Des niveaux d'eau sont imposés aux limites marines du modèle hydrodynamique. Ces variations du niveau d'eau sont obtenues à partir du modèle global de marée DTU10 développé par DTU Space (Technical University of Denmark). Les 12 principaux constituants de la marée sont calculés sur une grille d'une résolution de 0.125 x 0.125 degrés (CHENG \& ANDERSEN, 2012). 


\section{XIV ${ }^{\text {èmes }}$ Journées Nationales Génie Côtier - Génie Civil \\ Toulon, 29 juin au $1^{\text {er }}$ juillet 2016}

\subsection{Validation et calibration du modèle hydrodynamique}

Le modèle hydrodynamique a d'abord été calibré au Port de l'Herbaudière (figure 1), par rapport aux prédictions de la marée astronomique du SHOM. Le modèle reproduit correctement le signal de marée prédite avec une erreur RMS de $15 \mathrm{~cm}$ sur environ 1 mois. Lors des mesures hydrodynamiques, la pression atmosphérique était normale (1013 hPa), le vent faible et la mer calme. Une surcote moyenne d'environ $20 \mathrm{~cm}$ a été mesurée au marégraphe du port de l'Herbaudière (REFMAR) pendant la campagne. Cette surcote est prise en compte de manière schématique en décalant le signal de marée aux limites du domaine de calcul. Le modèle a ensuite été calibré par rapport aux mesures de niveaux d'eau de l'ADV en ajustant le paramètre de rugosité du fond. Ainsi, une rugosité plus importante a été paramétrée sur l'ensemble de la Baie de Bourgneuf. Le modèle simule de manière satisfaisante le signal de la marée au moment du déploiement de l'ADV: les effets de seuil induits par l'ouvrage sont bien reproduits (figure 5, gauche). Le schéma de circulation des courants est aussi assez bien reproduit : on observe les deux axes principaux de circulation mais avec des vitesses globalement surestimées par le modèle (figure 5 , droite). Le premier axe suit celui de la chaussée (ESE-WNW), il correspond au phénomène de vidange et de remplissage par le chenal lorsque les niveaux d'eau sont plus bas que l'ouvrage; le deuxième axe est perpendiculaire au Gois (NNE-SSW), il correspond au courant de flot venant du Nord de la baie et au début du jusant portant vers le Nord qui traversent la chaussée lorsque les niveaux d'eau dépassent l'altitude de l'ouvrage.
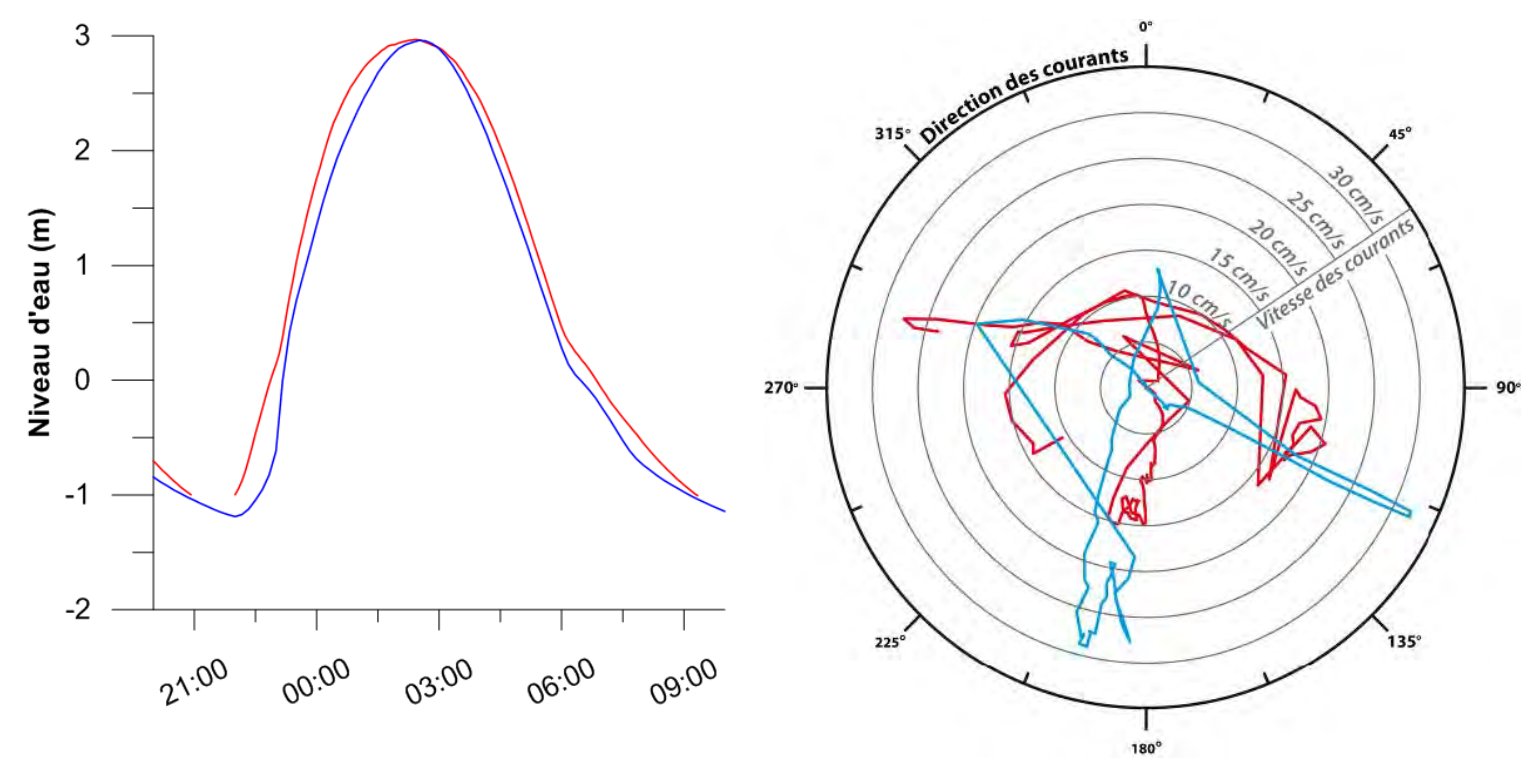

Figure 5. Comparaison de la simulation (en bleu) et des mesures (en rouge) des niveaux d'eau et de la circulation des courants au Passage du Gois. 


\section{Thème 2 - Dynamique sédimentaire}

\subsection{Résultats des simulations hydrodynamiques sur un cycle de marée}

La rencontre actuelle des courants de flot provenant de l'ouverture Sud et ceux provenant de celle du Nord est simulée à environ 1 à $2 \mathrm{~km}$ au Sud du Passage du Gois (figure 6). On peut se poser la question du lien entre la diminution de la section mouillée de l'embouchure tidale de Fromentine et le déplacement vers le Sud de la rencontre des deux courants.

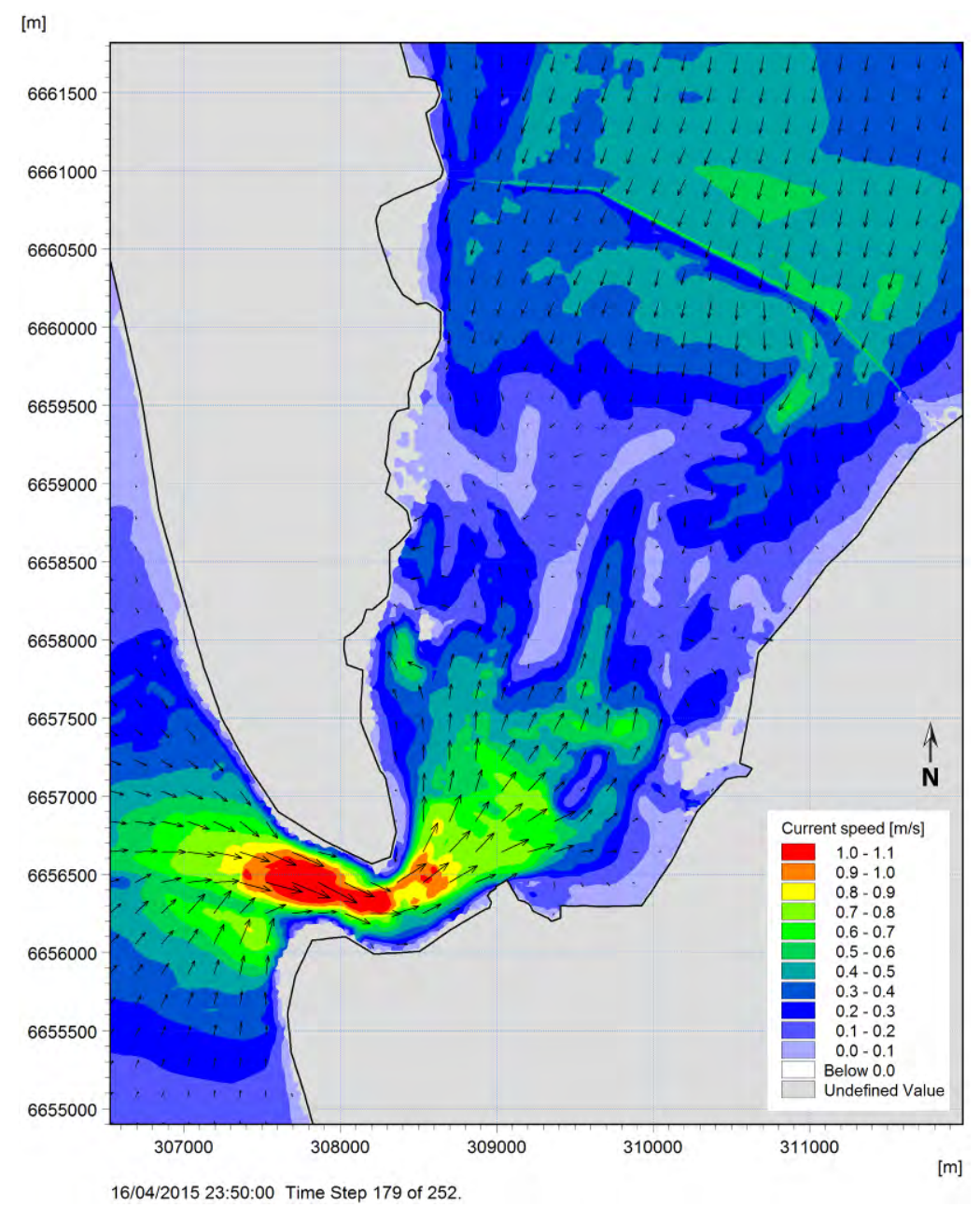

Figure 6. La convergence des flots de direction opposée dans le versant Sud de la Baie de Bourgneuf environ $2 \mathrm{~h}$ avant la pleine mer.

3.4 Résultats des simulations du transport solide sur un cycle de marée

Deux classes de grains ont été choisies à partir des cartes sédimentologiques du BRGM (BRGM, 2013) : $200 \mu \mathrm{m}$ et $1000 \mu \mathrm{m}$. Des tests de sensibilité sur la granulométrie des sables ont montré que les schémas de transport sont du même type pour les deux classes ; seule l'intensité des flux est modulée selon le diamètre du grain paramétré dans le modèle. 


\section{XIV èmes Journées Nationales Génie Côtier - Génie Civil \\ Toulon, 29 juin au $1^{\text {er }}$ juillet 2016}

La capacité de transport net est simulée sur une marée complète pour nous permettre de localiser les zones préférentielles d'érosion ou de dépôts. Les flux résiduels traversent globalement le Passage du Gois du Nord-Est vers le Sud-Ouest (figure 7). De tels résultats confirment, le long du bord Sud de la chaussée, une érosion liée au courant de flot telles que semblaient l'indiquer les évolutions morphologiques sur les images aériennes (figure 2) après la mise en place d'un ouvrage en dur. Au sud du Passage, pour certains tronçons, les courants de vidange ou de remplissage qui longent le chenal marquent localement les flux sédimentaires résiduels (figure 7).

Bien que la rencontre des deux flots de direction opposée s'effectue plus au Sud que le Gois, on ne constate pas de zone de convergence marquée de vecteurs témoignant d'un déplacement d'une zone d'accumulation sableuse plus au Sud (figure 7).

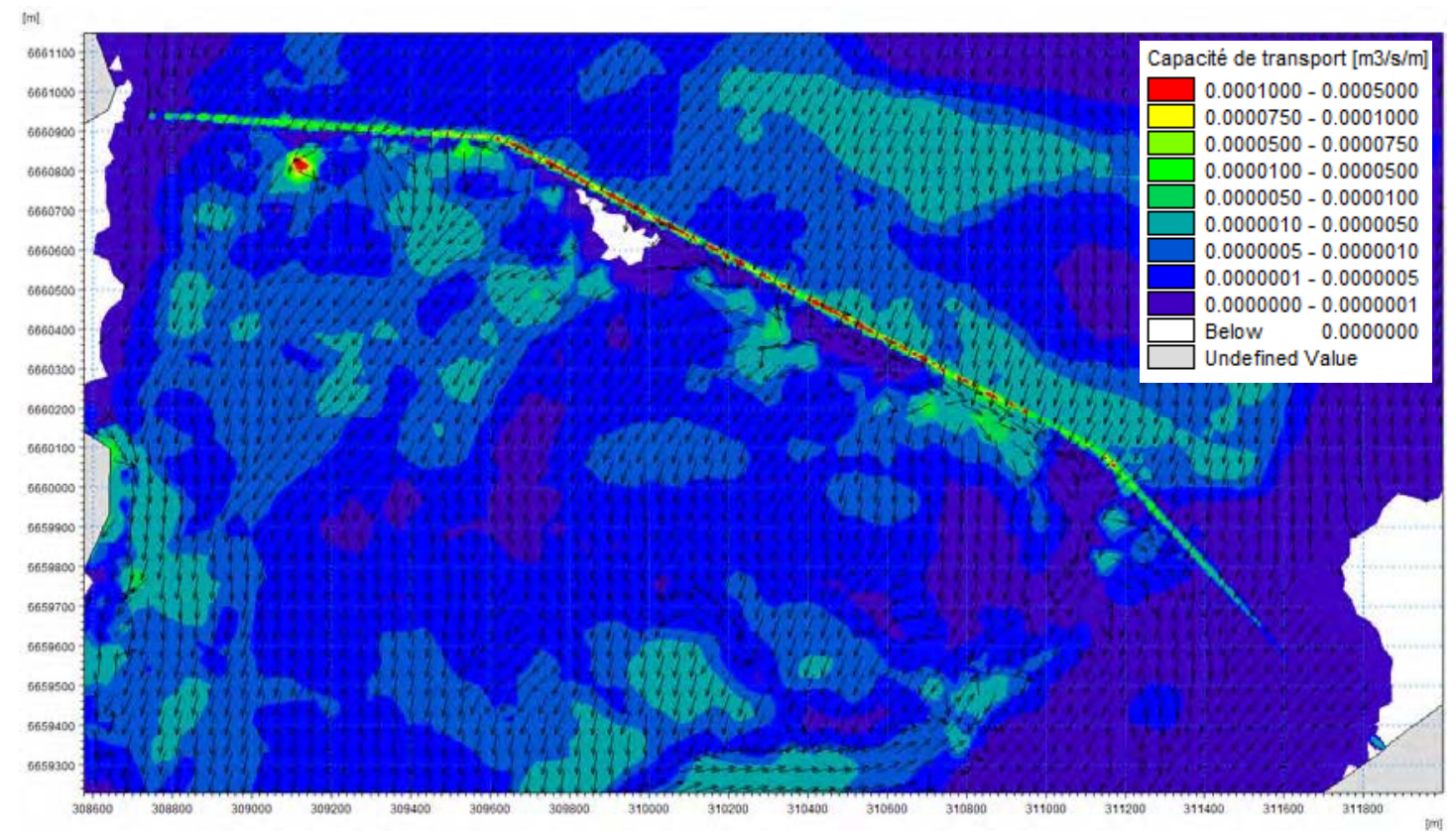

Figure 7. Capacité de transport résiduel pour une granulométrie à $200 \mu \mathrm{m}$.

\section{Conclusion.}

La modélisation hydro-sédimentaire a permis de préciser une partie des mécanismes à l’origine de l'érosion sur la partie Sud de la chaussée. La marée est le processus dominant dans la baie de Bourgneuf. Le courant de flot qui se propage dans la baie par l'ouverture Nord à chaque marée au travers du Passage du Gois du Nord-Est vers le Sud-Ouest, génère un transport résiduel dans le même sens. Ce flux sédimentaire se traduit par une érosion sur certains secteurs de la partie Sud de la chaussée. Cependant, certains processus restent à préciser, par exemple : dans des conditions de fort vent de secteur nord qui peut accélérer les courants de flot, dans quelle mesure les vagues peuvent augmenter la capacité de transport au travers du Passage du Gois. 


\section{Références bibliographiques}

BERNIER P., GRUET Y. (2011). Environnement littoral : sédimentation et biodiversité de l'estran, Ile de Noirmoutier (Vendée). Editions du département des sciences de la terre, Université Claude Bernard-Lyon 1, Villeurbanne, 163 p.

BRGM (2013). Cartographie géologique harmonisée du littoral des Pays-de-la-Loire. Rapport final BRGM/RP-62383-FR, 82 p.

CHENG Y., ANDERSEN O.B. (2012). Improvement of global ocean tide models in shallow water regions. Altimetry for Oceans and Hydrology, OST-ST Meeting, SV.16845.

COQUET M., MAANAN M., BOTREL J. (2014). L'évolution historique du Passage du Gois du $18^{\text {ème }}$ siècle à nos jours. Les Cahiers nantais, 2014-2, pp 17-24.

CREOCEAN (2011). Etude du fonctionnement hydro-sédimentaire et solutions de stabilisation du chenal de Fromentine. Rapport de la phase 2 : Analyse du fonctionnement hydro-sédimentaire du système, Conseil Général de Vendée.

O'BRIEN M.P. (1969). Equilibrium flow areas of inlets on sandy coasts. Journal of Waterways and Harbours Division, Vol. 95(1), pp 43-52.

SUIRE Y. (2011). La côte et les marais du Bas-Poitouvers 1700 : cartes et mémoires de Claude Masse, ingénieur du roi. Editions du Centre Vendéen de Recherches Historiques, La-Roche-sur-Yon, 366 p.

VERGER F. (2009). Zones humides du littoral français. Belin, Paris, 447 p. 